\title{
La Responsabilité Sociétale De L'entreprise : Les Différentes Approches Conceptuelles
}

\author{
Jihane Dinia (Doctorante) \\ Faculté des Sciences Juridiques, Economiques et Sociales - Agdal- \\ Mohammed V University in Rabat, Maroc \\ Abdellatif Chakor (Enseignant chercheur) \\ Faculté des Sciences Juridiques, Economiques et Sociales - Souissi- \\ Mohammed V University in Rabat, Maroc
}

Doi: 10.19044/esj.2018.v14n10p165 URL:http://dx.doi.org/10.19044/esj.2018.v14n10p165

\begin{abstract}
What are we talking about when we refer to the concept of Corporate Social Responsibility (CSR)? Behind every word that constitutes "CSR" lie great debates, starting with its definition. The purpose of our article is to emphasize the issue of the CSR through a brief genealogical overview and the confrontation of several definitions based on an academic literature review. Our study revealed a proliferation of proposed definitions of the concept, reflecting different approaches, which certainly enriched the debate, but complicated the subject. Therefore, after 50 years of research, CSR remains a very subjective concept, which is not yet complete..
\end{abstract}

Keywords: Corporate social responsibility, stakeholders, sustainable development, genealogy

\section{Résumé}

De quoi parle-t-on lorsqu'on évoque le concept de Responsabilité Sociétale des Entreprises ? Derrière chaque mot qui constitue la «RSE»se cachent des grands débats, à commencer par sa définition. L'objectif de notre article est de mettre l'accent sur la problématique du concept RSE à travers un bref survol généalogique puis la confrontation de plusieurs définitions émanant d'une revue de littérature académique. Notre étude a révélé une prolifération des définitions proposées du concept, traduisant des approches différentes, qui certes enrichissent le débat, mais complexifient le sujet. Il s'avère donc qu'après 50 ans de recherche, la RSE demeure un concept très subjectif, qui n'est pas encore abouti.

Mots clés : RSE, parties prenantes, développement durable, généalogie 


\section{Introduction}

Depuis plusieurs années jusqu’à nos jours, « la responsabilité sociétale des entreprises » dite RSE demeure un sujet majeur faisant débat dans les milieux académiques et scientifiques. Toutefois, en dépit du grand nombre des travaux de recherche qui se sont intéressés à ce sujet, la définition du concept ne fait pas l'unanimité ni au niveau de ses fondements théoriques ni de ses implications sociétales et organisationnelles. Ce constat est corroboré par les travaux d'Allouche, Huault et Schmidt (2004) qui ont souligné de nombreuses confusions entourant la définition de la RSE, entretenues par les différents acteurs : chercheurs, institutions... Toutefois, la multitude de définitions formulées par la littérature, ou par plusieurs organismes, indique que chacun l'entame d'un point d'ancrage différent en se référant à une discipline pour le cerner. Gond et Maullenbach-Servayre (2003), ont souligné que le concept de Responsabilité Sociale des Entreprises a des contours qui ont évolué au fil du temps.

Issue du concept d'origine anglo-saxonne de Corporate Social Responsibility, la RSE présente certaines ambigüités en termes d'interprétation. D'une part, ses diverses traductions en langue française sont loin de faire l'unanimité. D'autre part, selon Igalen et Gond (2008), cela s'explique par les différences à la fois culturelles, idéologiques et socioéconomiques entre les contextes français et anglo-américain.

Tandis que la notion anglaise « social » dont le sens managérial renvoie aux relations qu'entretient l'entreprise avec, aussi bien ses diverses parties prenantes directes (Stakeholders ou $\mathrm{SH}$ ), que la société dans son ensemble; sa version francophone se restreint à considérer exclusivement la nature des relations de travail entre employeur et employé. Cette vision conçoit l'entreprise dans la représentation classique de la firme comme une entité fermée dans un environnement stable. La gestion interne des relations employeur/employé est la seule préoccupation des dirigeants (en plus de la production des richesses). Ainsi, la notion «sociétale » vise à pallier l'interprétation restreinte et mieux incarner la réalité sociale des entreprises. L'entreprise est « socialement » responsable envers ses seuls salariés mais elle est « sociétalement » responsable envers les autres parties prenantes en plus de ses employés.

Dans notre travail, le qualificatif « social» sera retenu dans son sens large anglo-saxon, qui correspond au terme «sociétal » selon l'approche francophone.

L'objectif du présent article est de mettre l'accent sur la problématique du concept RSE à travers la confrontation de plusieurs définitions émanant d'approches différentes. Dans un souci d'enrichir la réflexion, nous présenterons, dans un premier temps une brève généalogie du concept avant 
de focaliser l'attention sur l'évolution historique de ses définitions fournies par la littérature académique.

\section{Brève généalogie du concept du RSE}

Le concept de RSE a une histoire longue et variée. Cette notion trouve ses origines aux États-Unis depuis la fin du $19^{\text {ème }}$ siècle. Néanmoins, certains lui revoient des antécédents à partir du temps de l'Athènes classique (500 ans av. J.-C.).

Jadis en Grèce Antique, la croyance voulait que, les individus qui étaient aisés financièrement et avaient du pouvoir ou de la puissance, soient socialement responsables. La notion « héroïsme » désignait certains aspects de responsabilité sociale et le terme grec «héros» se référait d'abord spécialement aux guerriers, mais plus tard, aux individus qui ont œuvré pour la communauté locale. Par ailleurs, certains travaux de la littérature managériale montrent que la RSE, sous sa forme élémentaire, date des années 30 et 40 .

L'idée que l'entreprise doit s'intéresser aux dimensions sociétales de son environnement s'ancre dans un ensemble de débats et de réflexions plus anciens. Il s'agit des travaux de Bernard (1938) «the Fonctions of the Exécutive », des articles fondateurs de Clark (1939) «Social Control of Business » et de Kreps (1940) «Mesurement of Social Performance of Business » (cité par Carroll 1999).

Ce n'est qu'aux années 50 que l'économiste universitaire Howerd Bowen (1953), que l'on considère comme le père fondateur du concept, a publié son ouvrage "Social Responsabilities of the Businessman ». Désormais, il est devenu la référence majeure de tout chercheur sur la RSE; et la recherche académique s'est progressivement structurée autour de grandes problématiques. Il s'agit de la définition du concept (Mc Guire, 1963), la mise en perspective de ses différentes dimensions (Sethi, 1975), l'opérationnalisation et la mise en évidence de ses liens avec la performance (Drucker, 1984), ou encore son exploration au travers du prisme offert par différents courants théoriques (approche parties prenantes, éthiques des affaires, etc.).

Parmi les adeptes du concept, on cite Carroll, Davis K., Frederick W.C, Bolstron, et Freeman. Quant à ses adversaires, il s'agit principalement de Friedman et Levitt. Un bref retour sur l'histoire est nécessaire pour assurer une certaine compréhension de ce concept et des pratiques y associées. 


\section{Naissance du concept RSE aux Etats-Unis avec l'apparition de la « Grande entreprise »}

La question des préoccupations sociétales est de mise depuis plusieurs décennies dans toutes les parties du monde par de grandes entreprises se souciant des besoins de leur personnel. Même si les années 50 sont considérées comme la naissance de la RSE aux Etats-Unis, certains auteurs ont identifié la fin du $19^{\text {ème }}$ siècle comme point raisonnable pour dater ses origines.

En effet, la fin du $19^{\text {ème }}$ siècle, pendant l'ère industrielle, était marquée par le paternalisme et la charité des grands capitalistes humanistes et d'inspiration religieuse qui prenaient en charge certains des besoins fondamentaux de leur personnel. Ainsi, l'approche paternaliste et l'aspect caritatif des hommes d'affaires sont les fondements de cette notion.

Les travaux incluant une dimension historique de ce concept montrent que l'idée de la RSE s'est diffusée en Amérique dans les milieux d'affaires et de la société dans un contexte de transformation du capitalisme américain (Epstein, 2002 ; Heald, 1961, 1970 ; Miller et O’Leary, 1989).

Pendant le $19^{\text {ème }}$ siècle, les entreprises s'apparentaient à ce que l'on appelle aujourd'hui, les compagny towns. Elles avaient un rôle prépondérant dans la vie de leurs employés, du fait qu'elles détenaient la propriété de toute l'infrastructure de la ville, notamment celle de la santé, de l'éducation et de l'approvisionnement. Face à une telle situation, l'Etat providence s'est édifié et le champ d'action des entreprises est devenu plus focalisé sur les initiatives de « philanthropie ».

Au début du $20^{\text {ème }}$ siècle, le débat s'est structuré autour de la question de la RSE de manière plus formalisée ${ }^{1}$ (Acquier et Gond, 2005), avec l'apparition d'un nouveau modèle d'institution « la grande entreprise ». Selon Miller et O’Leary (1989), les premières théories de management (Taylor, Follett, Mayo, et Barnard) ont toutes contribué à rendre «socialement acceptable » cette nouvelle organisation en légitimant le principe de hiérarchie qui en est indissociable. Il parait que l'acceptation et la légitimation de cette forme d'entreprises étaient les véritables enjeux de l'émergence de la RSE.

Or, les caractéristiques de cette nouvelle institution - l'actionnariat dispersé, la figure du dirigeant salarié non propriétaire et la professionnalisation du management de l'entreprise - ont constitué un terrain particulièrement favorable pour la diffusion de l'idée de la RSE (Epstein, 2002 ; Heald 1970).

${ }^{1}$ Ces premières formulations sont centrées sur la conscience individuelle des dirigeants, et se sont particulièrement marquées par l'importance accordée à la philanthropie et à une redistribution des richesses via des donations des riches aux plus défavorisés (Zunz, 2005). Cette tradition philanthropique renvoie au mouvement d'évangélisation de la sphère sociale ou Social Gospel (cf. Carnergie) 
La séparation entre la propriété et la gestion, dans cette nouvelle forme d'entreprises, est désormais une caractéristique qui a imposé une diminution du contrôle exercé auparavant par les actionnaires sur les dirigeants. L'entreprise est devenue une entité à part entière et a commencé à s'institutionnaliser, en raison de sa taille et de l'éloignement de ses propriétaires de la direction. De ce fait, elle apparait de plus en plus redevable vis-à-vis d'un ensemble de « groupes intéressés, incluant cette vague entité, la communauté dans son ensemble » (Heald, 1961). L'aspect de la RSE est ici apparent dans la question de gouvernance de cette forme d'entreprise.

Quant à la professionnalisation du management, elle a donné lieu à l'émergence d'une nouvelle profession et une nouvelle figure du dirigeant. Pour de telles raisons, des Business schools américaines ont été mises en place; ainsi que des programmes de formation permettant de développer la sensibilité des dirigeants aux effets de leurs décisions sur le fonctionnement large de la société. De plus, cette dernière caractéristique de l'entreprise a permis de transformer le capitalisme familial qui avait prévalu précédemment dans les entreprises, qui commençaient à recruter des ingénieurs et des juristes formés dans ces Business Schools américaines. La RSE reflète l'émergence d'une déontologie des dirigeants et de nouvelles normes professionnelles.

En reprenant les propos de Bowen, Acquier et Aggeri, (2007), la notion RSE s'est diffusée au départ dans les champs d'affaires portés par des hommes d'affaires de l'époque et centrés sur la figure du dirigeant plutôt que sur l'entreprise (Henry Ford, Alfred Sloan, Chester Barnard ou encore les dirigeants de la General Electric Company).

A partir des années 20, plusieurs dirigeants se sont exprimés sur leur responsabilité envers la société, par leurs discours à connotation religieuse de « sevice public » et de «trusteeship ». Ces derniers stipulent un contrat implicite caractérisant la relation entre l'entreprise et la société.

De nouvelles actions philanthropiques et de mécénat (Heald 1961, 1970) des grandes entreprises se sont développées au cours de cette période, accompagnées par des négociations collectives et la mise en place des démarches de communication-relations publiques (Marchand 1998). Mais, elles étaient mises en veille pendant la crise de 1929, ce qui a suscité une grande dépression et une méfiance importante du grand public vis-à-vis des entreprises. Par conséquent, la RSE était atteinte par l'interventionnisme public et la coercition (Pasquero, 2005).

Toutefois, la participation de ces entreprises à la seconde guerre mondiale, était l'occasion pour qu'elles restaurent leur prestige auprès de la société américaine, et s'expriment à nouveau publiquement sur leur responsabilité sociale. Après 1945, la notion de RSE a resurgi comme un véritable vecteur de légitimation des entreprises et du système capitaliste, et les discours à son propos sont devenus acceptables voire à la mode. 


\section{Formalisation du concept à la lumière de la réflexion de Bowen}

Les années 50 ont connu le début de la théorisation du concept voire même l'émergence d'une « doctrine de Responsabilité sociale » qui a vu le jour à la frontière entre théologie, économie et gestion. L'ouvrage théorique de Bowen (1953) «Social Responsibilities of the Businessman »était le premier produit des efforts significatifs dans ce sens.

L'auteur d'obédience religieuse chrétienne, notamment, protestante a structuré son témoignage historique autour des notions de stewardship, de trusteeship et de prise en compte du bien-être social. En 1953, il a ouvert la réflexion sur la RSE, fondée sur une hypothèse structurante qui est la subordination de l'entreprise et de la sphère économique à la société.

Les réflexions de Bowen sont apparues dans une période de transformation de l'économie américaine, ou de sa sortie du laissez faire. Il décrivait 1'économie américaine des années 50 de «mixed economy », c'està-dire, une économie à mi-chemin entre le libéralisme et le socialisme. Selon Bowen, s'ils veulent s'évincer d'un système plus encadré et proche du socialisme qui limitait leur liberté d'action, les dirigeants d'entreprise devaient « atteindre un équilibre raisonnable entre l'intérêt privé et public » (Acquier, Aggeri, 2007). Donc, la RSE doit passer par l'intégration des valeurs recherchées globalement par les composantes de la société, au-delà des seuls objectifs économiques poursuivis par les actionnaires et des obligations légales qui contraignent leurs décisions.

En fait, pour sa religion protestante, la recherche du profit ne peut être l'ultime fin de l'entreprise privée. L'objectif de servir l'intérêt public et accroitre le bien-être de la société doit être aussi important que la réalisation des bénéfices. Dans ce sens, les dirigeants des entreprises devaient, avec une reconsidération de leurs actions et implications sociales, chercher à converger à terme les intérêts de l'entreprise avec ceux de la société.

Les années 60 représentaient une décennie où le débat portant sur la RSE a connu un véritable essor. Le fait que les comportements et les activités des entreprises étaient loin du respect des droits de l'homme, de l'environnement, des consommateurs, etc., ils sont devenus sujets à des mouvements civiques, consuméristes et écologistes qui les contestaient.

A ce propos, Acquier et Aggeri (2008) ont cité les exemples de Ralph Nader qui a remis violemment en cause les pratiques des constructeurs automobiles (en particulier General Motors) et leur acharnement à éviter l'introduction de normes de sécurité dans leurs produits à travers sa publication «Unsafe at Any Speed 》 (Nader, 1965) ; ainsi que Carson qui a évoqué, pour les problèmes environnementaux, les dégâts irrémédiables dus à l'usage des pesticides par certaines entreprises, et qui a remis en cause l'idée selon laquelle l'environnement a une capacité d'absorption infinie. 
Sur le plan institutionnel, les préoccupations environnementales ont été traduites par l'adoption du Clean Air Act en Californie en 1963, du Wilderness Act en 1964 et la création de l'Agence pour la protection de l'environnement (EPA) au début des années 1970. En réaction aux mouvements consuméristes, l'agence publique de protection des consommateurs, la Consumer Product Commission (CSPC), et l'Amnesty International ont été créées en 1961.

Pour le droit du travail, une commission américaine sur l'égalité des opportunités d'emplois (US Equal Employment Opportunity Commission) a été mise en place en 1964. Finalement, la création dans les années 1970 de l'Occupational Safety and Health Administration pour la question de la sécurité au travail qui a fait aussi l'objet de réglementation.

Les pressions de différentes démarches activistes se sont traduites par la réglementation des comportements des entreprises et leur soumission à un cadre législatif contraignant et coûteux. Cette approche coercitive (command and control) a transformé le capitalisme américain et c'est une forme de RSE obligatoire qui est apparue. De nombreux dirigeants engageaient leurs entreprises soit par obligation morale, soit le plus souvent en vue de se préserver une marge de manœuvre suffisante face à l'Etat et aux groupes de pressions populaires (enlightened self-interest).

\section{Développement de concepts intégrateurs}

A partir des années 80 , c'est l'époque de consolidation des construits théoriques de la RSE et de ses grandes définitions fondatrices. Les auteurs s'attachent à conceptualiser la RSE et ses champs d'action pour l'entreprise.

La vague "éthique » de la RSE héritée du paternalisme d'entreprise du $19^{\text {ème }}$ siècle, et fondée sur les valeurs morales et religieuses depuis les années 50 avec le livre de Bowen (1953) a laissé la place au développement d'une autre conception de la RSE. A partir des années 70 et durant les années 80 et 90 , le concept de RSE s'est détaché des exigences "éthique » et «morale » à l'égard de la société. On commence à le reformuler en termes stratégiques.

La nouvelle conception de la RSE a mis l'accent sur les exigences économiques de l'entreprise. Du point de vue de celle-ci, le comportement social de l'entreprise doit servir sa performance économique. C'est l'approche « utilitariste » de la RSE.

Cette nouvelle orientation de la recherche a donné lieu à la naissance de nouveaux termes d'investigations théoriques. Il s'agit de :

- La notion de «performance sociale de l'entreprise » qui concerne le lien entre performance économique et performance sociale de l'entreprise ;

- La notion des «Parties prenantes (PP)» évoquée par Freeman (1984). Selon l'auteur, la prise en compte par l'entreprise des attentes de ses 
actionnaires, ses salariés et autres parties contractantes, voire au-delà, toute partie qui est susceptible d'affecter ses intérêts, est aussi incontournable que décisive lors du développement de ses stratégies ;

- La notion de « réactivité sociale de l'entreprise » qui fait référence au processus de mise en œuvre de stratégie RSE. C'est un courant développé dans le cadre d'un travail de recherche au sein de la Harvard Business School en 1971 sous l'autorité de Raymond Bauer (Ackerman, 1975). Ce concept renvoie à la capacité des firmes multidivisionnelles à piloter le changement et à renouveler leurs modes d'action de manière régulière, au-delà de la capacité des entreprises à prendre en charge de nouvelles questions sociales ;

- La notion de «business case » qui repose sur une stratégie gagnantgagnant, centrée sur la nécessité économique pour l'entreprise de se conformer aux attentes sociétales pour garantir sa présence dans le marché et sa profitabilité. Les enjeux associés à la RSE deviennent le gain de rentabilité, l'avantage concurrentiel, l'image de marque, etc.(Capron \& Petit, 2011).

A partir des années 90, un autre courant en lien avec le développement durable (DD) émerge. Il s'agit du courant « soutenable » de la RSE dont les enjeux sont ceux de la contribution de l'entreprise au DD.

Alors qu'aux Etats-Unis, la RSE est resté très libérale et empreinte du caractère religieux protestant, dans le reste du monde, notamment, en Europe, ce concept s'est développé d'une façon très institutionnelle, évoquant le concept du développement durable (DD).

\section{Internationalisation du concept}

Bien que la dernière décennie du $20^{\text {ème }}$ siècle ait connu un foisonnement des thématiques de RSE étudiées aux Etats-Unis, sa prise en compte concrète, en tant qu'approche volontaire par les entreprises, date de la fin des années 90 .

En fait, la propagation du concept hors d'Amérique est liée à plusieurs raisons. Il s'agit d'une part, de l'ouverture des marchés globaux et les nouveaux défis de la globalisation, des critiques de la société civile à l'encontre du modèle libéral, des conséquences du développement technologique et économique (biogénétique, nucléaire, internet, réchauffement climatique). D'autre part, sa prise en compte au niveau international découle d'une évolution législative (loi française sur les nouvelles régulations économiques de 2002, directives européennes sur l'emballage, lois de grenelle, etc.) et de la montée concomitante du concept de DD depuis le rapport de Bruntland (1987).

Dans ce cadre général, et face à l'affaiblissement des Etats, la question d'une RSE se posait avec insistance comme à la fois nécessaire et durable. Klaus Schwab, fondateur du forum économique mondial indiquait que la réunion du forum de 1999 à Davos «constitua peut être un moment décisif 
pour la réintroduction de la notion de responsabilité sociale dans le monde des entreprises » (cité dans Banlanyà et al., 2000, p149).

Dans ce sens, de grandes organisations internationales, telles que l'Organisation de Coopération et Développement Economiques (OCDE), l'Union Européenne (UE) et l'Organisation des Nations Unies (ONU) ont participé, à leur tour, au mouvement d'intégration des entreprises dans les thématiques de RSE et du DD. Elles ont développé leurs propres approches « institutionnelles » de la RSE.

\section{L'approche RSE de l'OCDE}

En 1976, l'OCDE a proposé une série de «Principes directeurs pour les entreprises multinationales » construits dans une vision positive de la mondialisation économique. Ces principes servaient d'instruments facilitateurs de l'investissement direct entre les membres de l'OCDE (OCDE, 2001b). Pour cette organisation, l'investissement international est le fer de lance de la mondialisation dont il s'agit désormais de gérer pour en partager les bénéfices; et les multinationales sont les canaux par lesquels il passe pour se concrétiser. $\mathrm{Vu}$ « la contribution positive que les entreprises multinationales peuvent apporter au progrès économique, environnemental et social » (ibid., p.3), on les considère comme partie intégrante de l'économie internationale. Elles sont une source des flux d'investissement, des recettes fiscales et de la diffusion de technologies. Toutefois, leurs activités ne cessent de susciter certaines inquiétudes chez l'opinion publique, ce qui justifie la nécessité de leur cadrage. Les principes directeurs sont une réponse en ce sens. Ce sont « des principes et des normes volontaires pour un comportement responsable des entreprises dans plusieurs domaines » (ibid., p.2).

Dans ce contexte, chaque multinationale devait s'y référer comme instrument lui permettant d'opérer en harmonie avec le pays où elle est située, et d'empêcher les malentendus.

En 2000, l'OCDE a révisé ses principes proposés en 1976, acte qui a permis l'intégration de nouvelles exigences en matière d'environnement, de droit de l'homme, de travail (suppression du travail des enfants et le travail forcé), de lutte contre la corruption, et de protection du consommateur. C'était un renforcement des "éléments essentiels - économique, social, et environnemental - du programme d'action du développement durable » (ibid., p.4).

Le réexamen effectué met en évidence l'intérêt que porte l'OCDE au terme DD. D'ailleurs, dans l'une de ses définitions de la notion de RSE, elle se réfère exclusivement au concept de DD : « la RSE est la contribution des entreprises au développement durable » (OCDE 2001a, p 13, n.t).

Dans le même sens, l'OCDE considère qu'on " s'entend en général pour dire que les entreprises dans une économie globale sont souvent appelées 
à jouer un plus grand rôle au-delà de celui de création d'emplois et de richesses; et que la RSE est la contribution des entreprises au développement de la durabilité ; que le comportement des entreprises doit non seulement assurer des dividendes aux actionnaires, des salaires aux employés et des produits et services aux consommateurs, mais il doit répondre également aux préoccupations et aux valeurs de la société et de l'environnement » (Voir : www.oecd.org/home).

Dans cette définition, l'OCDE met l'accent sur le rôle crucial que doit jouer l'entreprise dans le DD. Tout en cherchant à maximiser son profit et à créer de la richesse et de l'emploi, l'entreprise peut réussir à préserver son environnement et à respecter les valeurs de sa société. Tel est le défi que les entreprises sont appelées à relever en se référant aux principes volontaires de l'OCDE, et en s'engageant dans une démarche RSE.

\section{L'approche RSE de la Commission Européenne}

Bien qu'une batterie de définitions institutionnelles potentielles soit disponible, celle que la Commission Européenne $(\mathrm{CE})^{2}$ est la plus souvent utilisée.

Elle définit la RSE comme "L'intégration volontaire par les entreprises de préoccupations sociales et environnementales à leurs activités commerciales et leurs relations avec les PP» (Livre Vert, juillet 2001, p.8). La définition nous éclaire sur deux convictions de la CE : l'engagement de l'entreprise dans la démarche RSE ne peut être que volontaire et doit s'appuyer sur un objectif de DD. Le concept de RSE couvre, au-delà, des domaines sociétal et environnemental, la réponse de l'entreprise aux attentes et aux besoins de ses PP.

Ce document de référence était destiné, d'une part, à déclencher le débat sur le concept de RSE et, d'autre part, à définir les moyens de construire un partenariat permettant l'élaboration d'un cadre tant européen qu'international pour la promotion du concept.

La CE s'est appuyée sur les conclusions du Conseil Européen de Lisbonne de mars 2000, qui mettent l'accent particulièrement sur la RSE, durant lequel l'UE s'est fixée un objectif stratégique pour la première décennie du $21^{\text {ème }}$ siècle. Cet objectif s'exprime en termes d'économie la plus compétitive et la plus dynamique du monde, de croissance durable, d'amélioration de l'emploi et de cohésion sociale.

Il en résulte l'appui à l'investissement du champ de la RSE par l'UE. Il s'agit d'une stratégie en faveur du DD qui encourage les entreprises à élargir leurs champs de RSE : "l'action des pouvoirs publics est (...)

${ }^{2}$ Définition présentée dans son Livre vert « Promouvoir un cadre européen pour la responsabilité sociale des entreprises » 
essentielle pour encourager les entreprises à davantage prendre conscience de leurs responsabilités sur le plan social et pour mettre en place un cadre permettant de s'assurer que les entreprises intègrent les aspects environnementaux et sociaux dans leurs activités (...) il faudrait encourager les entreprises à intégrer de manière active le développement durable dans les activités qu'elles poursuivent à l'intérieur de l'UE et dans le monde " (CE, 2001c, p.8).

Un an plus tard, la consultation des porteurs d'enjeux et l'avis des institutions européennes ont amené la CE à publier sa «Communication concernant la RSE : une contribution des entreprises au développement durable " (CE, 2002). L'objectif était de préciser que la notion RSE est intimement liée au concept du DD. Constat qu'a expliqué le président de la $\mathrm{CE}$, Romano Prodi, par l'évolution de la prise de conscience et des attentes des citoyens européens. Les exigences de ceux-ci vont, désormais, au-delà de rémunérations justes, des produits de bonnes qualités aux bons prix, à d'autres types de demandes portant sur des valeurs éthiques et plus généralement sur le DD.

\section{L'approche RSE de l'ONU}

En 1999, inquiet de la fragilité de la globalisation par la prédominance de l'économique sur le politique et le social, Kofi Annan le secrétaire général des NU, a proposé aux entreprises de promouvoir un ensemble de valeurs universelles et les intégrer volontairement à leurs actions. En fait, il s'agit de l'extension de la responsabilité sociale de l'entreprise et l'intégration de ces valeurs dans les champs des droits de l'homme, du travail et de l'environnement.

Le Global Compact (GC) ou le Pacte Mondial, lancé officiellement en Juillet 2000, s'inscrit dans cette lignée. Cet outil est le fruit du discours de Kofi Annan dont l'objectif était la mise en place d'un «Pacte mondial » afin d'avoir des valeurs partagées au niveau du marché global. Dès lors, l'ONU s'est engagée dans l'édiction des principes de RSE auxquels les entreprises sont invitées à adhérer. Son rôle était l'assistance et la facilitation du dialogue entre les entreprises et les autres groupes sociaux sans pour autant avoir un pouvoir contraignant. L'initiative d'adhésion découle d'une démarche volontaire des entreprises en s'engageant à respecter dix principes ayant pour objectifs la défense desdits droits et la lutte contre la corruption ajoutée en 2004 (Encadré $1)$. 
Encadré 1 : Les dix principes du Global Compact

Principe 1 : Encourager et respecter la protection des droits de l'homme internationaux dans sa sphère d'influence.

Principe $2:$ S'assurer que sa propre entreprise ne soit pas complice de violations des droits de l'homme. Principe 3 : Liberté d'association et reconnaissance effective du droit à la négociation collective.

Principe 4 : Elimination de toutes formes de travail forcé et obligatoire.

Principe 5 : Abolition effective du travail des enfants.

Principe 6 : Elimination de la discrimination dans l'embauche et le travail.

Principe 7 : Encourager une approche des défis environnementaux fondée sur le principe de précaution. Principe 8 : Mettre en œuvre des initiatives pour promouvoir une plus grande responsabilité environnementale.

Principe 9 : Favoriser le développement et la diffusion.

Principe 10 : La lutte contre la corruption

Source : https://www.unglobalcompact.org/what-is-gc/mission/principles

Le GC n'est pas une norme ou un code de conduite. Il relève d'une charte de principes qui impliquent un certain engagement des pays adhérents. En s'engageant, ces pays doivent s'efforcer à mettre la charte en œuvre et à promouvoir son respect par les entreprises opérant dans ou à partir de leur territoire. Toutefois, les entreprises elles-mêmes ne sont pas tenues d'adhérer publiquement aux principes, alors qu'elles le doivent si elles veulent adhérer au GC.

Par ailleurs l'engagement des entreprises au GC se déroule encore très humblement. Certains expliquent cela par la méfiance qu'éveillent en particulier les principes concernant le travail. (Elkington, 2001)

En définitive, on peut remarquer que les principes de l'OCDE et de l'ONU présentent des instruments globaux de responsabilité des entreprises, qui servent de standards de référence aux entreprises internationales en matière de performance et pratiques non financières. Par contre, l'approche de l'UE se trouve en plein débat instigué par son livre vert. Celui-ci ne fixait pas des méthodes pour le développement de la RSE, mais il amorçait la recherche des outils permettant de constituer des piliers d'une politique européenne de promotion de la RSE.

Par ailleurs, l'approche onusienne insiste aussi clairement que celle de l'OCDE sur le caractère volontaire et non contraignant de son instrument non prescriptif. A la différence, le livre vert et la communication de la CE mettent l'accent sur les questions de suivi et de contrôle des engagements des entreprises.

L'objectif de cette brève lecture généalogique était de cerner l'identité du concept de RSE, et de comprendre le contexte historique de sa naissance 
aux États-Unis et de sa diffusion dans d'autres contextes culturels. Toutefois, l'internationalisation du concept RSE ne signifie guère sa standardisation, car bien que des principes universels semblent se dégager (principes de l'OCDE ou initiatives de l'ONU et ses agences), il reste nécessaire de les adapter aux besoins du pays qui s'en empare.

Force est de constater la diversité des approches et des définitions de la RSE proposées par les différentes institutions. Quid de celles données par les académiciens?

\section{Littérature académique}

Comme il a été montré ci-dessus, le concept de RSE a une histoire longue et variée. Toutefois, la temporalité académique ne coïncide pas avec celle de la société. Les discours académiques qui suivent ne sont qu'un prolongement d'un ensemble de pensées qui se sont développées depuis les années 50 avec Bowen. Plusieurs autres chercheurs l'ont succédé et ont tenté de le définir selon différentes perceptions sans aboutir à un consensus (Davis, 1960 ; McGuire, 1963 ; Jones, 1980 ; Friedman, 1962 ; Manne, 1972 ; Preston et Post, 1975 ; Carrol, 1979 ; Watrick et Cochran, 1985 ; Wood, 1991 ; Clarkson, 1995 ; Husted, 2000).

Toutes ces recherches et d'autres ont abouti au développement de différentes approches du concept RSE. Certaines ont considéré que la RSE va au-delà de l'intérêt économique de la firme, d'autres l'ont réduite à la maximisation du profit. Tandis qu'un groupe de chercheurs a avancé que la RSE consiste à répondre aux attentes de la société de façon volontaire, et finalement un autre groupe a mis l'accent sur le fait que la RSE se traduit par la prise en compte des intérêts de ses PP.

\section{La RSE va au-delà de l'intérêt économique de la firme}

Pour Bowen, premier auteur ayant défini le concept, la RSE « renvoie à l'obligation pour les hommes d'affaires, de mettre en ouvre les politiques, de prendre les décisions et de suivre les lignes de conduite qui répondent aux objectifs et aux valeurs considérés comme désirables pour notre société » (Bowen, 1953). Dans cette définition, l'auteur souligne l'impératif de la contribution de l'entreprise à la réalisation des objectifs de sa société.

Selon cette approche, il serait plus judicieux de parler de Responsabilité Sociétale des Entreprises, car le terme "sociétale » renvoie à la société dans son ensemble, alors que le terme «social» fait uniquement référence à la politique sociale adoptée par l'entreprise pour satisfaire les besoins de ses salariés comme il a été précisé précédemment.

Pour l'entreprise, prendre en compte les conséquences de ses actions sur la société implique que la nature de ses responsabilités augmente. L'entreprise ne doit plus avoir des objectifs purement économiques, comme 
le suggère le courant libéral, mais également des préoccupations sociales. La RSE va donc au-delà de l'intérêt économique de la firme.

Plusieurs autres universitaires américains se sont intéressés audit concept et ont cherché à donner leurs propres acceptions. Peter Drucker (1954), a consacré une partie de son ouvrage « the practice of management », à la responsabilité des managers. Selon l'auteur, les intérêts économiques de l'entreprise et les enjeux sociaux sont indissociables. Druker rejette la vision utilitariste smithienne de l'économie selon laquelle une quête " aveugle » de la maximisation de profits conduit à un bien-être collectif. Son approche consiste à éclairer les managers sur la nécessité de prendre conscience des enjeux relatifs à la manière de conduire les affaires.

D'autres chercheurs sont allés dans la même veine de Bowen. Davis (1960) est l'un des auteurs les plus évoqués dans ce sujet. Lors de sa définition de la notion RSE, il a mis en évidence la dimension éthique du terme. Pour lui, «La RSE est la considération de la firme et sa réponse à des problèmes qui vont au-delà des engagements économiques, techniques et légaux pour atteindre le seuil du bénéfice social » (Davis 1960). Néanmoins, selon cet auteur, les décisions et actions socialement responsables ne manquaient pas d'avantages économiques sur le long terme de l'activité des entreprises qui s'y engageaient. Il a maintenu sa conviction, lorsqu'il a affirmé avec Blomstrom que « la responsabilité sociale se rapporte à l'obligation d'une personne de considérer les effets de ses actions dans l'ensemble du système social. Les dirigeants appliquent la RSE quand ils considèrent les besoins et les intérêts d'autres personnes qui pourraient être touchées par les actions de l'entreprise. Ainsi, ils regardent au-delà des intérêts économiques et techniques étroits de leur entreprise » (Davis et Blomstrom 1966, cité à par Carroll, 1999).

De même, Frederick (1960) a défendu que les moyens de production devaient être utilisés de telle sorte qu'ils améliorent le bien être socioéconomique total (Carroll, 1999). Sur la même lancée, McGuire (1963) a avancé que l'idée de responsabilité sociétale suppose que la firme n'a pas seulement des obligations légales ou économiques, mais elle les dépasse à des obligations sociétales.

D'autres auteurs à l'instar de Jones (1980) et McWilliams et Siegel (1997) ont considéré, également, que la RSE est une responsabilité extraéconomique. Selon les approches de ces auteurs, une entreprise a, par dessus ses obligations économiques et légales à respecter, des engagements sociétaux à honorer envers la société dans laquelle elle évolue.

Toutefois, d'autres auteurs ont rejeté cette idée en rappelant que la raison d'être de l'entreprise est la réalisation de profit. 


\section{La RSE consiste à maximiser le profit pour les actionnaires}

L'auteur de référence de l'approche selon laquelle l'entreprise doit avoir des préoccupations économiques pures, est Milton Friedman. Ce partisan du courant libéral, s’opposant aux idées du livre de Bowen (1953), a décrit la notion de RSE comme, profondément subversive. Il considérait que «Rien n'est plus dangereux, pour les fondements de notre société, que l'idée d'une responsabilité sociétale des entreprises autre que de générer un profit maximum pour leurs actionnaires » Friedman (1962). Lors de sa publication «Freedom and capitalism » (1962), il a affirmé que « la seule responsabilité de l'entreprise est de maximiser les profits de ses propriétaires et actionnaires ».

De même en 1970, Friedman a confirmé cette approche en avançant que «La responsabilité sociétale de l'entreprise est celle d'accroitre ses profits. Elle consiste à utiliser ses ressources et à s'engager dans des activités destinées à accroitre ses profits, dans la mesure où elle respecte les règles $d u$ jeu, c'est-à-dire celles d'une concurrence ouverte et libre ». D'après cet auteur, les dirigeants ne sont que des mandataires des actionnaires. Ils ont des responsabilités envers les propriétaires qui les emploient dans le sens où ils doivent mener le travail comme ceux-ci le désirent. Ce travail se réduit à maximiser le profit autant que possible, tout en respectant les règles légales et éthiques.

Sa position est sans ambiguïté : « si les hommes d'affaires ont une responsabilité autre que le maximum de profit pour les actionnaires, comment peuvent-ils savoir ce qu'elle est ? Des individus privés auto-désignés, peuventils décider ce qu'est l'intérêt de la société et se substituer à l'État ?» (Friedman, 1970, cité par Gond, p. 335).

C'est une approche compatible avec une vision classique de la firme où la rémunération des actionnaires est légitime et justifiée. L'entreprise ne peut pas avoir des responsabilités sociétales; seules les personnes telles que les dirigeants en ont. Ces derniers peuvent assumer personnellement et volontairement certaines responsabilités envers la société mais sans engager leur entreprise. Ils peuvent donc agir pour leur propre compte et dépenser leur propre argent. Mais, en aucun cas, ils ne peuvent agir au nom de leurs employeurs et juger à leur place pour un intérêt social d'ordre général.

Pour Friedman, ces actions ne seraient admises que dans le cas où elles sont accompagnées d'une économie d'impôt pour l'entreprise, sinon, elles risquent de contrecarrer sa recherche du profit, ce qui va à l'encontre des principes d'une économie libérale.

La RSE consiste à répondre aux attentes de la société de façon volontaire

Contrairement aux auteurs qui ont mis l'accent sur les obligations sociétales de l'entreprise, d'autres chercheurs ont développé une autre 
approche selon laquelle la réponse de l'entreprise aux attentes de la société doit être volontaire et non pas obligatoire. Il s'agit notamment de Walton (1967), dans son ouvrage « Corporate social responsabilities », où la notion de " volontarisme » a été reconnue pour la première fois. Cet auteur précisait que la RSE d'une entreprise comprenait toujours un certain degré de volontarisme, car les entreprises devaient accepter que des coûts soient impliqués dans des actions sociales sans retour économique mesurable. (Walton, 1967, cité par Carroll, 1999).

Dans un sens similaire, Manne (1972) a souligné que la RSE est l'idée selon laquelle les firmes répondent aux attentes de la société de façon volontaire. Pour sa part, Jones (1980) a avancé que «La RSE n'est en aucun cas une obligation de nature coercitive. L'entreprise est tenue d'adopter un comportement responsable, mais toute action sociale influencée par une contrainte légale n'est en aucun cas volontaire ».

D'après ces auteurs, l'engagement sociétal de l'entreprise doit traduire sa pleine volonté d'assumer cette responsabilité. La RSE ne doit pas être conçue par l'entreprise comme une obligation ou une contrainte mais comme un choix stratégique.

L'étude de cette approche volontariste de la RSE ne peut être évoquée sans citer les travaux de Carroll considéré, aux Etats-Unis, comme le père du sens actuel du concept. Selon cet auteur, la responsabilité sociétale est ce que la société attend, à un moment donné, des organisations en matière économique, légale, éthique et volontaire (Carroll 1979). Cette conception est présentée dans son modèle des quatre catégories de la responsabilité sociale de l'entreprise. (Voir Figure 1).

Figure 1 : Les 4 catégories de la RSE selon le modèle de Carroll de 1979

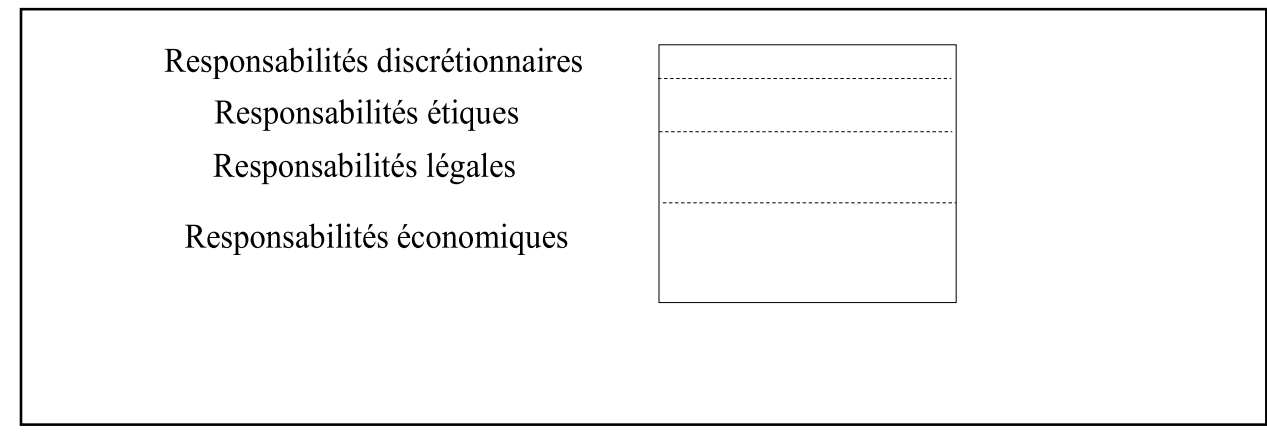

Source : Carroll 1979

Carroll considérait que les responsabilités des entreprises envers la société peuvent être regroupées dans les quatre catégories de son modèle dont la proportion de chacune donne une idée sur l'importance accordée à la responsabilité en question. 
On remarque que les responsabilités étalées dans le modèle de Carroll (1979) sont présentes toutes à la fois dans le monde des affaires et que la possibilité qu'une action d'entreprise puisse avoir en même temps les quatre objectifs économiques, légaux, éthiques et/ou discrétionnaires, est aussi présente. Toutefois, Carroll a précisé que l'histoire économique montre que la proportion de la dimension économique est la plus importante suivie de la dimension légale. Les aspects éthiques et discrétionnaires viennent respectivement au $3^{\text {ème }}$ et au $4^{\text {ème }}$ rang.

En 1991, Carroll a repris son modèle initial de 1979 pour développer une nouvelle conceptualisation de la RSE sous la forme d'une pyramide (voir Figure 2).

Figure 2 : Les 4 catégories de la RSE selon le modèle de Carroll de 1991

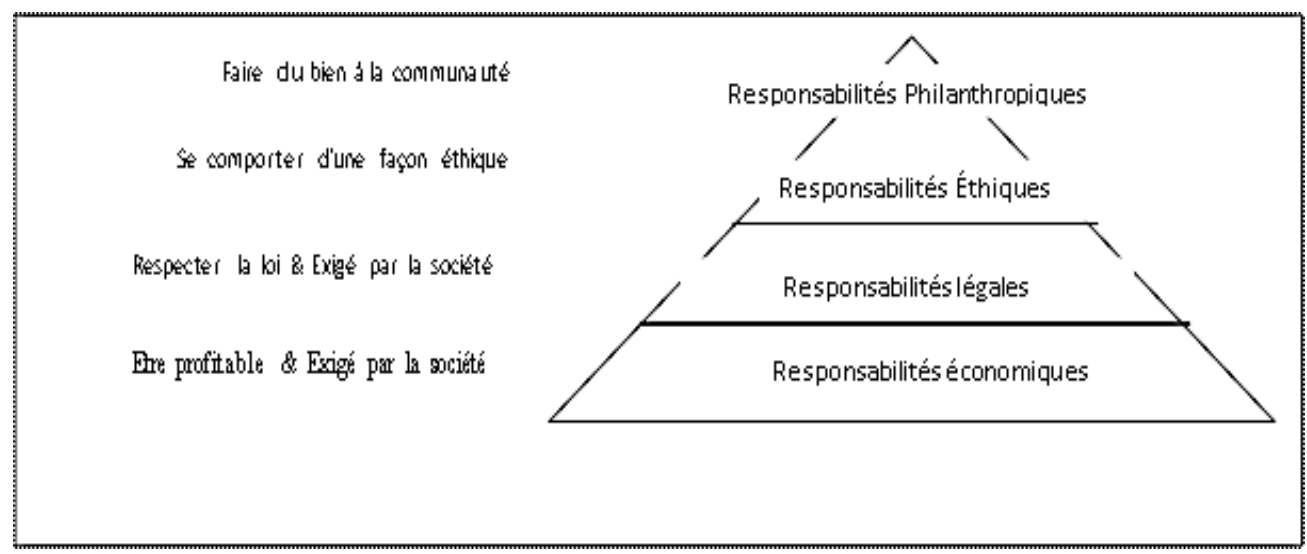

Source : Carroll 1991

Etant donné que la raison d'être de l'entreprise est la réalisation du profit, les principales actions réalisées par cette organisation, en termes de quantité et qualité, sont de type économique. Par ailleurs, si l'entreprise ne joue pas convenablement ce rôle qui lui est assigné par la société, elle ne pourra pas être un agent actif sur le plan social. Il est donc tout à fait normal que les responsabilités économiques soient la base de la RSE. Le respect de la loi, $2^{\mathrm{ème}}$ responsabilité de l'entreprise, est fondamental en matière de RSE vu que les lois représentent un processus de codification des valeurs morales qui sont présentes dans la société (Crane et Matten, 2004).

Selon Carroll (1991), les responsabilités éthiques, classées en $3^{\text {ème }}$ position, obligent les entreprises à faire ce qui est vu comme bon, juste et honnête. Il s'agit des actions que les PP attendent d'une entreprise même si elle n'y est pas tenue par la loi. L'engagement d'une entreprise dans une démarche RSE atteint son apogée, lorsqu'elle réalise volontairement des actions désirées par la société pour le bien-être de ses salariés et/ou de la communauté en général: il s'agit des responsabilités philanthropiques. Le fait 
que ces dernières siègent au sommet de la pyramide veut dire qu'elles sont moins présentes dans ses préoccupations.

Dans cette perspective, les responsabilités s'ajoutent les unes aux autres et chacune d'entre elles forme un composant de base de la responsabilité sociétale totale d'une entreprise.

Bien que le modèle de Carroll ait été utilisé à plusieurs reprises dans les recherches aussi bien théoriques qu'empiriques sur la RSE, il présente néanmoins un certain nombre de limites. Ces dernières ont poussé son auteur à le revoir en 2003 pour ne garder que trois niveaux de responsabilités en fusionnant les deux derniers qui semblent être très proches (Responsabilités éthiques et philanthropiques).

De même, Wood (1991) a revu et complété la définition de Carroll, en précisant trois niveaux de responsabilités pour l'entreprise :

la responsabilité de l'entreprise en tant qu'institution sociale : la Société accorde une légitimité à l'entreprise. En contrepartie, dans l'exercice de son pouvoir économique découlant de cette légitimité, l'entreprise doit répondre aux attentes la Société ;

la responsabilité en terme de conséquences de ses activités : l'entreprise est responsable des conséquences de son activité sur ses parties prenantes premières (concernées directement par les décisions de la firme) ou secondaires (concernées indirectement) ;

- la responsabilité individuelle et morale des dirigeants qui doivent intégrer la RSE aussi bien lors de la conception de leurs stratégies que lors de leur mise en œuvre. (Attarça \& Jacquot, 2005)

Ainsi, selon Wood 1991 «La responsabilité sociétale ne peut être appréhendée qu'à travers l'interaction de trois principes : la légitimité, la responsabilité publique, et la discrétion managériale. Ces principes résultent de trois niveaux d'analyse institutionnel, organisationnel et individuel ».

L'approche de Wood est complémentaire à celle de Carroll. Chacune des formes de responsabilité proposées par Carroll (économique, légale, éthique et philanthropique) peut être déclinée selon les trois niveaux définis par Wood. Le tableau $n^{\circ} 1$ qui suit fait un rapprochement des modèles de Carroll et de Wood en croisant les niveaux et la nature de la RSE: 
Tableau $\mathrm{N}^{\circ} 1$ : Niveau et nature de la RSE

\begin{tabular}{|c|c|c|c|}
\hline & $\begin{array}{c}\text { Niveau } \\
\text { Institutionnel }\end{array}$ & $\begin{array}{c}\text { Niveau } \\
\text { Organisationnel }\end{array}$ & $\begin{array}{c}\text { Niveau } \\
\text { individuel }\end{array}$ \\
\hline $\begin{array}{c}\text { Responsabilités } \\
\text { Economiques }\end{array}$ & $\begin{array}{l}\text { Produire des biens et } \\
\text { services; offrir des } \\
\text { emplois ; créer de la } \\
\text { richesse pour des } \\
\text { actionnaires. }\end{array}$ & \begin{tabular}{|c|} 
Les prix des biens et \\
services reflètent les \\
vrais coûts de production \\
et intègrent toutes les \\
externalités.
\end{tabular} & $\begin{array}{c}\text { Produire de façon } \\
\text { écologique, utiliser des } \\
\text { technologies non } \\
\text { polluantes, réduire les } \\
\text { coûts en favorisant le } \\
\text { recyclage. } \\
\end{array}$ \\
\hline $\begin{array}{c}\text { Responsabilités } \\
\text { Légales }\end{array}$ & $\begin{array}{c}\text { Respecter les lois et les } \\
\text { réglementations. } \\
\text { Ne pas faire de lobbying } \\
\text { ou attendre des positions } \\
\text { privilégiées dans les } \\
\text { politiques publiques. }\end{array}$ & \begin{tabular}{|c|} 
Euvrer pour des \\
politiques publiques en \\
défendant des intérêts \\
éclairés.
\end{tabular} & $\begin{array}{l}\text { Tirer profits des } \\
\text { instructions } \\
\text { réglementaires pour } \\
\text { innover dans les } \\
\text { produits ou les } \\
\text { technologies. } \\
\end{array}$ \\
\hline $\begin{array}{c}\text { Responsabilités } \\
\text { Ethiques }\end{array}$ & $\begin{array}{l}\text { Suivre des principes } \\
\text { Ethiques fondamentaux } \\
\text { (par exemple } \\
\text { l'honnêteté). }\end{array}$ & \begin{tabular}{|c|} 
Fournir des \\
Informations précises et \\
complètes pour accroitre \\
la sécurité d'utilisation \\
au-delà des conditions \\
légales. \\
\end{tabular} & \begin{tabular}{|c|} 
Développer \\
l'information \\
d'utilisation pour des \\
usages spécifiques et la \\
promouvoir comme un \\
avantage produit. \\
\end{tabular} \\
\hline $\begin{array}{c}\text { Responsabilités } \\
\text { Discrétionnaires }\end{array}$ & $\begin{array}{l}\text { Agir comme un citoyen } \\
\text { modèle dans tous les } \\
\text { domaines : au-delà des } \\
\text { réglementations et des } \\
\text { règles éthiques, rendre } \\
\text { une partie du chiffre } \\
\text { d'affaires à la société } \\
\text { (Community). }\end{array}$ & $\begin{array}{l}\text { Investir les Ressources } \\
\text { de l'entreprise dans des } \\
\text { actions charitables en } \\
\text { rapport avec le premier } \\
\text { et le second cercle de } \\
\text { l'environnement sociale } \\
\text { de l'entreprise. }\end{array}$ & $\begin{array}{c}\text { Choisir des } \\
\text { investissements } \\
\text { charitables qui soient } \\
\text { rentables en termes de } \\
\text { résolution de } \\
\text { problèmes sociaux } \\
\text { (application de critères } \\
\text { d'efficacité). }\end{array}$ \\
\hline
\end{tabular}

Source : Adapté du modèle de Carroll et Wood (1991)

\section{La RSE consiste à tenir compte des intérêts de ses Parties Prenantes}

Toutes les responsabilités évoquées précédemment s'appuient sur la théorie des Parties Prenantes (PP). Berle \& Means (1932) peuvent être considérés comme les pionniers de cette approche «stakeholder». Mercier (2004) rappelle que Berle et Means, en 1932, avaient déjà constaté le développement d'une pression sociale s'exerçant sur les dirigeants pour qu'ils reconnaissent leur responsabilité auprès de tous ceux dont le bien-être peut être affecté par les décisions de l'entreprise. Berle \& Means (1932) n'ont pas utilisé explicitement le terme de parties prenantes mais leurs réflexions ont notablement évoqué le concept. Pour Berle, les intérêts des différents partenaires seraient mieux servis par un attachement sans faille des dirigeants à la richesse des actionnaires.

Par ailleurs, la notion de PP a été évoquée pour la première fois avec Harold Johnson en 1971 dans son livre intitulée « Business in contemporary : framework and issues ». L'auteur considérait qu'une entreprise socialement 
responsable devait, d'une part, tenir compte de l'intérêt de ses employés, fournisseurs, revendeurs, et des collectivités locales. D'autre part, concilier entre des intérêts divers, au-delà, de l'intérêt de l'accumulation des richesses des actionnaires.

De son coté, Jones (1980) a défendu l'idée qu'une entreprise socialement responsable est celle qui rajoute, volontairement, les intérêts des autres (PP) à ses propres intérêts et les considère comme étroitement liés. Ainsi, il a défini la RSE comme " la notion selon laquelle les entreprises ont une obligation envers les groupes constitutifs de la société autres que les actionnaires et cela, au-delà de ce qui est prescrit par la loi ou le contrat syndical »

Carroll (1991) également a préconisé d'utiliser la notion de PP pour mieux identifier la RSE car l'entreprise est devenue soumise à de grandes pressions de la part des acteurs de sa société (les actionnaires, les employés, les clients, les fournisseurs, les lois et les réglementations, les groupements de la société civile ...).

Les attentes des PP doivent être entendues et recevoir une réponse qui fait partie de la stratégie RSE de l'entreprise, celle-ci devant inclure une gestion des relations avec les PP. Dans ce sens, la norme ISO 26000 inscrit la « prise en compte des PP » au cœur de la notion de RSE qui se définit comme la « maîtrise par une organisation des impacts de ses décisions et activités sur la société et sur l'environnement, se traduisant par un comportement éthique et transparent qui contribue au développement durable, y compris à la santé et au bien-être de la société, prend en compte les attentes des PP ; respecte les lois en vigueur tout en étant en cohérence avec les normes internationales de comportement; et qui est intégré dans l'ensemble de l'organisation et mis en ouvre dans ses relations ». Il n'est pas donné de liste de PP, celles-ci étant tout « individu ou groupe ayant un intérêt dans les décisions ou activités d'une organisation ».

L'approche des PP amène l'entreprise à isoler les groupes d'acteurs les plus « pertinents » et qu'il convient de considérer dans une démarche de RSE. Ceci est également perçu dans la définition d'Epstein (1987) selon laquelle la RSE est «la réalisation des résultats des décisions organisationnelles concernant les questions spécifiques qui ont des avantages plutôt que des effets indésirables sur les PP pertinentes d'entreprise ». Selon Epstein (1987), la RSE est la satisfaction des PP qui constitue une condition nécessaire, en parallèle avec la mise en œuvre des systèmes de mesures originaux et appropriés pour la réussite organisationnelle.

\section{Synthèse des principales approches de la RSE}

La multiplicité des approches théoriques du concept RSE explique les divergences remarquées dans ses définitions académiques. Le tableau $\mathrm{n}^{\circ} 2$ ci- 
après synthétise l'évolution historique du concept de la RSE selon les principales approches développées ci-dessus.

Tableau n ${ }^{\circ} 2$ : L'évolution historique du concept RSE selon les principales approches

\begin{tabular}{|c|c|}
\hline \multicolumn{2}{|r|}{ La RSE va au-delà de l'intérêt économique de la firme } \\
\hline Bowen 1953 & $\begin{array}{l}\text { La RSE renvoi à l'obligation de mettre en œuvre les politiques, de } \\
\text { prendre les décisions, et de suivre les lignes de conduite qui } \\
\text { répondent aux objectifs et aux valeurs considérées comme } \\
\text { désirables par la société. }\end{array}$ \\
\hline Davis 1960 & $\begin{array}{l}\text { La RSE est la considération de la firme et sa réponse à des } \\
\text { problèmes qui vont au-delà de ses engagements économiques, } \\
\text { techniques et légaux pour atteindre le seuil du « social benefit ». }\end{array}$ \\
\hline Mc Guire 1963 & $\begin{array}{c}\text { L'idée de responsabilité sociétale suppose que la firme n'a pas } \\
\text { seulement des obligations légales ou économiques, mais qu'elle } \\
\text { possède également des responsabilités envers la société, qui dépasse } \\
\text { le simple cadre de ces obligations. }\end{array}$ \\
\hline Backman 1975 & $\begin{array}{l}\text { La RSE renvoie aux objectifs et aux raisons qui donne une âme aux } \\
\text { affaires plutôt qu'à la recherche de la performance économique. }\end{array}$ \\
\hline Jones 1980 & $\begin{array}{c}\text { La responsabilité sociale est l'idée selon laquelle les firmes, au-delà } \\
\text { des prescriptions légales ou contractuelles, ont des obligations } \\
\text { envers les acteurs sociétaux. }\end{array}$ \\
\hline $\begin{array}{l}\text { Mc Williams et } \\
\text { Siegle } 2001\end{array}$ & $\begin{array}{c}\text { La RSE est l'ensemble des actions qui répondent aux attentes de la } \\
\text { société et qui vont au-delà des intérêts économiques de la firme } \\
\text { dans le respect des lois. }\end{array}$ \\
\hline \multicolumn{2}{|r|}{ La RSE consiste à maximiser le profit pour les actionnaires } \\
\hline Friedman 1962 & $\begin{array}{c}\text { Rien n'est plus dangereux pour les fondements de notre société que } \\
\text { l'idée d'une responsabilité sociétale des entreprises autre que de } \\
\text { générer un profit maximum pour leurs actionnaires. }\end{array}$ \\
\hline Frien & $\begin{array}{l}\text { La responsabilité sociétale de l'entreprise est celle d'accroitre ses } \\
\text { profits. Elle consiste à utiliser ses ressources et à s'engager dans des } \\
\text { activités destinées à accroitre ses profits, dans la mesure où elle } \\
\text { respecte les règles de jeu, c'est-à-dire celles d'une concurrence } \\
\text { ouverte et libre. }\end{array}$ \\
\hline \multicolumn{2}{|c|}{ La RSE consiste à répondre aux attentes de la société de façon volontaire } \\
\hline Manne 1972 & $\begin{array}{l}\text { La responsabilité sociétale est l'idée selon laquelle les firmes } \\
\text { répondent aux attentes de la société de façon volontaire. }\end{array}$ \\
\hline Caroll 1979 & $\begin{array}{l}\text { La responsabilité sociétale est ce que la société attend à un moment } \\
\text { donné des organisations en matière économique, légal, éthique et } \\
\text { volontaire. }\end{array}$ \\
\hline Jones 1980 & $\begin{array}{l}\text { La RSE n'est en aucun cas une obligation de nature coercitive. } \\
\text { L'entreprise est tenue d'adopter un comportement responsable, } \\
\text { mais toute action sociale influencée par une contrainte légale n'est } \\
\text { aucun cas volontaire. }\end{array}$ \\
\hline Frederick 1994 & $\begin{array}{l}\text { L'acceptation volontaire des principes de responsabilité est toujours } \\
\text { préférable à la réglementation ou à l'intervention contraignante. }\end{array}$ \\
\hline \multicolumn{2}{|c|}{ La RSE consiste à tenir compte des intérêts de ses Parties Prenantes } \\
\hline $\begin{array}{c}\text { Maignan, Ferrell } \\
\text { et Huit, } 1999\end{array}$ & $\begin{array}{l}\text { La RSE est la mesure dans laquelle les entreprises assument les } \\
\text { responsabilités économiques, légales, éthiques et discrétionnaires } \\
\text { imposées par les diverses parties prenantes aux activités de } \\
\text { l'entreprise }\end{array}$ \\
\hline
\end{tabular}




\begin{tabular}{|c|c|}
\hline Gendron 2002 & $\begin{array}{c}\text { La responsabilité sociale concerne l'ensemble des relations que la } \\
\text { firme entretient avec ses parties prenantes. Les éléments de la } \\
\text { responsabilité sociale incluent l'investissement dans la } \\
\text { communauté, les relations avec les employés, la création et le } \\
\text { maintien de l'emploi, les préoccupations environnementales et la } \\
\text { performance financière. }\end{array}$ \\
\hline $\begin{array}{c}\text { Luo et } \\
\text { Bhattacharya, } \\
2006\end{array}$ & $\begin{array}{c}\text { Le concept de RSE renvoie aux activités et engagements de } \\
\text { l'entreprise liés à la perception de ses obligations envers la société } \\
\text { ou les parties prenantes à son activité }\end{array}$ \\
\hline
\end{tabular}

Source : Elaboré par nos propres soins à partir des travaux de Carroll (1999); Gond $\S$

Mullenbach (2004); V. Swaen § R. Chupitaz (2008) et FE. Taoufik (2015)

\section{Conclusion}

La lecture généalogique du concept RSE montre qu'il s'est profondément transformé au fil du temps selon les conditions de son émergence. Si au début il était plutôt lié aux valeurs morales et religieuses, il s'est enuite enrichi d'une dimension éthique. Puis, il s'est imposé comme l'outil qui permet d'aborder les défis du capitalisme et de mondialisation. Enfin, il a poursuivi son évolution en lien avec le développement durable.

Eu égard à son importance, le concept de RSE a attiré l'attention de plusieurs organisations de nature diverses : des ONG, des institutions européennes et internationales. Toutefois, même si la formulation de sa définition est tributaire des objectifs propres à chaque institution, toutes les définitions institutionnelles mettent l'accent sur l'approche volontaire du concept, et la notion du DD, avec la prise en compte des intérêts des PP de l'entreprise.

Par ailleurs, la revue de littérature a révélé une prolifération des définitions proposées du concept, traduisant des approches différentes, qui certes, enrichissent le débat mais complexifient le sujet.

Au terme de ce travail, il s'avère qu'après 50 ans de recherche, la RSE demeure un concept très subjectif, qui n'est pas encore abouti. Au contraire, il est un processus en cours d'évolution, perçu de différentes façons selon le courant de pensée ou de l'idéologie de l'auteur.

\section{References:}

1. Acquier A. et Aggeri F. (2007), « Aux sources de la responsabilité sociale de l'entreprise : à la (re)découverte d'un ouvrage fondateur, Social Responsibilities of the Businessman d'Howard Bowen ", Finance Contrôle Stratégie - Volume 10, n 2, p. 5 - 35 .

2. Acquier A., Gond J.P. (2005). " Aux sources de la Responsabilité Sociale de l'Entreprise : relecture et analyse d'un ouvrage séminal : Social Responsibilities of the Businessman d'Howard Bowen (1953) ", XIVème Conférence de l'Association internationale de management stratégique (AIMS), Angers. 
3. Allouche J., I, Huault et G. Schmidt, (2004), « Responsabilité sociale des entreprises : la mesure détournée ? ", 15 ème Congrès annuel de l'AGRH, Montréal

4. Attarça M., Jacquot T. 2005, « La représentation de la responsabilité sociale des entreprises une confrontation entre les approches théoriques et les visions managériales "XIVème Conférence de l'Association internationale de management stratégique (AIMS), Angers.

5. Backman, J. (1975), « Social responsibility and accountability », New York, University Press.

6. Ben Yedder, M., \& Zaddem, F. (2009), « La Responsabilité Sociale de l'Entreprise (RSE), voie de conciliation ou terrain d'affrontements? ». Revue multidisciplinaire sur l'emploi, le syndicalisme et le travail, 4(1), 84-103.

7. Capron M. et Quairel-Lanoizelée F., (2007), «La responsabilité sociale d'entreprise », Paris, La Découverte, coll.Repères.

8. Cahier de la Chaire de responsabilité sociale et de développement durable : Gendron C. (2002), « Envisager la responsabilité sociale dans le cadre des régulations portées par les Nouveaux mouvements sociaux économiques», Chaire de responsabilité sociale et de développement durable, Montréal, École des sciences de la gestion, UQAM, no 012002.

9. Carroll A. (1979), «A Three-Dimensional Model of Corporate Performance ", Academy of Management Review, Vol. 4, No. 4, p. 497-505.

10. Carroll A. (1991), « The Pyramid of Corporate Social Responsibility: Toward the Moral Management of Organizational Stakeholders "), Business Horizons, Vol. 34, N4, p. 39-48.

11. Carroll A.B. (1999), « Corporate Social Responsibility. Evolution of a Definitional Construct », Business \& Society, vol. 38, n 3, p. 268-295.

12. Clarkson, M. B. E. (1995), «A stakeholder framework for analysing and evaluating Corporate Social Performance ", Academy of Management Review, 20:1, 92-117.

13. Commission Européenne, (2001), «Promouvoir un cadre européen pour la RSE», Livre vert, 366, Bruxelles, 31 p.

14. Commission Européenne, (2002), «la responsabilité sociale des entreprises : une contribution des entreprises au développement durable », 347.

15. Crane, A. \& Matten, D. (2004), «Business Ethics: A European Perspective - Managing Corporate Citizenship and Sustainability in the Age of Globalization», Oxford, Oxford University Press. 
16. Davis, K. (1960), « Can business afford to ignore social responsibilitie ? », Academic Management Review, 16.

17. Davis K. (1973), « The Case for and against Business Assumptions of Social Responsibilities », Academy of Management Journal, vol. 16, $\mathrm{n}^{\circ} 2$, p. 312-322.

18. Epstein E.M. (2002), «The Field of Business Ethics in the United States : Past, Present and Future [1] », Journal of General Management, vol. 28, $\mathrm{n}^{\circ}$ 2, p. 1-29.

19. Freeman R.E. (1984), «Strategic Management : A Stakeholder Approach», Pitman Publishing, Inc.

20. Friedman, M. (1962), «Capitalism and Freedom», Chicago, University of Chicago Press.

21. Friedman, M. (1970), « The social responsibility of business is to increase its profits», New York Times Magazine, 13, p. 32-33, 122, 124.

22. Gendron C. (2000), «Enjeux sociaux et représentations de 1'entreprise », La Revue du MAUSS, 15, 320-325.

23. Global Compact 2001, An Employers'Guide on the $G$

24. Gond, J.-P., Maullenbach-Servayre, A. (2003), « Les fondements théoriques de la responsabilité sociale de l'entreprise », Revue des Sciences de Gestion, ${ }^{\circ}$ 205, pp. 93-116.

25. Husted, B.W. (2000), « A contingency theory of corporate social performance», Business and Society, vol. 39, $\mathrm{n}^{\circ} 1$, p. 24-48.

26. Heald M. (1961), « Business Thought in the Twenties : Social Responsibility », American Quarterly, vol. 13, n 2, p. 126-139.

27. Heald M. (1970), The Social Responsibilities of Business : Company and Community, 1900-1960, Case Western Reserve University Press.

28. Jones, T.M. (1980), « Corporate social responsibility revisited, redefined », California Management Review, vol. XXII, n², p. 59-67.

29. J. Igalen et JP. Gond (2008), «La responsabilité sociale de l'entreprise », Collection Que sais-je, Ed PUF.

30. Manne H.G. \& Wallich, H.C. (1972), « The modern corporation and social responsibility » Washington, DC: American Enterprise Institute for Public Policy Research.

31. McGuire, J. (1963), "Business and Society », New York, McGrawHill

32. Mcwilliams, A. \& Siegel, D. (1997), «The role of money managers in assessing corporate social responsibility research», Journal of Investing, Winter, p. 98-107.

33. McWilliams \&Donald Siegel, (2001), «Corporate Social Responsibility: a Theory of the Firm Perspective», Academy of Management Review, Vol. 26, No.1, p 117-127. 
34. Miller P. et O'Leary T. (1989), " Hierarchies and American Ideas,1900-1940 », Academy of Management Review, vol. 14, n² 2, p. 250-265.

35. OCDE (2001), « Survey of Investment Regulations of Pension Funds ", Secretariat Paper Financial Affairs Department, Paris

36. OCDE, 2001b, «Synthèse : les Principes directeurs de l'OCDE à l'intention des entreprises multinationales", http://www.oecd.org/FR/document/0,,FR-document-93nodirectorate-no-14-5931-28,FF.html - consulté en mars 2002.

37. Pasquero J. (2005), «La responsabilité sociale de l'entreprise comme objet des sciences de gestion : un regard historique », in M.F. Turcotte

38. Persais, E. (2007). « La RSE est-elle une question de convention? ». Revue française de gestion, 33(172), p 79.

39. Preston 1. \& Post J.E. (1975), «Private management and Public Policy : The principals of publics responsibility », New Jersey, Englewood cliffs prentice-Hall.

40. Rodié, I. (2007). "Responsabilité sociale des entreprises. Le développement d'un cadre européen ", Mémoire présenté pour l'obtention du Diplôme d'études approfondies en études européennes, Institut Européen de l'université de Genève.

41. Taoukif, F. E. Z. (2014). "Analyse perceptuelle des déterminants de l'engagement sociétal des entreprises marocaines labellisées RSE: de la performance au développement durable-cas du Maroc », thèse pour l'obtention de doctorat en Sciences de gestion, l'Université Moulay Ismail de Meknès/ l'Université du Sud Toulon-Var.

42. Taleb, B. (2013). «Les motivations d'engagement des entreprises dans la responsabilité sociale : le cas du secteur industriel algérien ", thèse pour l'obtention de doctorat en Sciences de gestion, École doctorale $\mathrm{n}^{\circ} 372$ Sciences Économiques et de Gestion d'AixMarseille.

43. Wartick, S.L. \& Cochran, P.L. (1985), «The evolution of the corporate social performance model», Academy of Management Review, vol. 10, p. 758-769. 